\title{
Total Knee Replacement Delayed With Hylan G-F 20 Use in Patients With Grade IV 0steoarthritis
}

\author{
David D. Waddell, MD, and DeWayne C. Bricker, PA-C
}

\begin{abstract}
BACKGROUND: Total knee replacement (TKR), a last resort for treating knee pain due to osteoarthritis $(O A)$, is not always medically indicated or preferred by many patients. Hylan G-F 20 is a cross-linked hyaluronan derivative approved for the treatment of pain due to $\mathrm{OA}$ of the knee after other conservative approaches have failed.

OBJECTIVE: The objectives of this study were to (1) determine the effect of hylan G-F 20 on patient need for TKR as measured by time from hylan G-F 20 injection to TKR, and (2) assess patient factors that might influence time from hylan G-F 20 therapy to TKR.

METHODS: This is a retrospective case series review of the medical records of patients seen in 1 orthopedic specialty practice. The incidence and time to TKR in patients who were TKR candidates ( $100 \%$ grade IV OA [severe]) treated with 1 or more courses of intra-articular hylan G-F 20 injections (3 weekly injections per course) were determined from 0ctober 1997 to November 2003. Survival analysis was used to evaluate time to TKR and the effects of age, gender, ethnicity, body mass index (BMI), and presence of effusion on this outcome. Logistic regression was also used to assess these covariates.
\end{abstract}

RESULTS: The incidence of TKR in hylan G-F 20 -treated knees (1,187 knees; 863 patients) was $19 \%(n=225$ knees). The median time to TKR in these patients was 638 days (1.8 years; minimum of 14 days, maximum of 2,147 days). For patients in whom a TKR had not yet occurred during the observation time, the median time of hylan G-F 20 treatment and patient follow-up was 810 days (2.2 years; minimum of 7 days, maximum of 2,222 days). A total of 1,978 courses of hylan G-F 20 given to 1,187 knees (average 1.67 courses per knee) resulted in an average cost of $\$ 1,419.76$ per knee to delay TKR by a median of 2.1 years ( 772 days, minimum 7 , maximum 2,222), the median time of all knees to either TKR or time of last observation. Survival analysis showed that $75 \%$ of knees had not had a TKR by 1,370 days (3.8 years). Survival analysis and logistic regression indicated that of age, gender, ethnicity, BMI, and presence of effusion, only age significantly affected time to TKR.

CONCLUSION: In patients who are candidates for TKR, the need for TKR can be delayed with hylan G-F 20 when used for the treatment of $O A$ knee pain.

KEYWORDS: Hylan G-F 20, Knee, Osteoarthritis, Pain, Total knee replacement J Manag Care Pharm. 2007;13(2)113-21

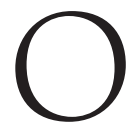
steoarthritis (OA) and other rheumatic conditions are the leading cause of disability in the United States. ${ }^{1}$ The prevalence of OA of the knee in various countries can be estimated as $18 \%$ of men and women as inferred by a recent meta-analysis. ${ }^{2} \mathrm{OA}$ can also result in significant direct and indirect costs, which are higher with greater disability. ${ }^{3}$

Current therapeutic options for OA are approved to treat the pain associated with the disorder. The American College of Rheumatology recommends the initial use of nonpharmacologic techniques, such as patient education, weight loss, and physical therapy, followed by pharmacologic therapies such as acetaminophen and cyclooxygenase (COX)-2-specific inhibitors. ${ }^{4}$ Intra-articular hyaluronan therapy is recommended for patients who are no longer responsive to nonpharmacologic and pharmacologic treatments or who may be experiencing adverse side effects with nonselective nonsteroidal inflammatory drugs or COX-2-specific inhibitors. ${ }^{4}$

Total knee replacement (TKR) is considered a last resort for treatment of $\mathrm{OA}$ knee pain in appropriate patients when other therapies fail. According to the National Hospital Discharge Survey published by the U.S. Department of Health \& Human Services, approximately 326,000 patients in the United States received a TKR in $2001 .^{5}$ In a sample of 6,856 decedents aged $\geq 65$ years, $2.1 \%$ were found to have received knee replacements. ${ }^{6}$ The cost of knee replacement surgery at patient discharge was reported as $\$ 24,045$ for $2001 .^{7}$

The success of TKR in most patients has been supported by more than 20 years of follow-up data. ${ }^{8}$ While TKR is an effective option for OA pain relief for many patients, it is not medically desirable for some patients (age, heart disease, diabetes, pulmonary disease, obesity, or generalized medical debilitation) or is not preferred by others. Additionally, complications of the

\section{Authors}

DAVID D. WADDELL, MD, is a clinical associate professor of orthopedics at Louisiana State University Health Sciences Center School of Medicine, Shreveport, $L A$, and an orthopedic surgeon affiliated with Orthopedic Specialists of Louisiana, Shreveport, LA; DEWAYNE C. BRICKER, PA-C, is a certified physician assistant, Marshall, Texas (at the time of this study, he was a certified physician assistant at Orthopedic Specialists of Louisiana, Shreveport, $L A$ )

CORRESPONDING AUTHOR: David D. Waddell, MD, Orthopedic Specialists of Louisiana, 1500 Line Ave., Shreveport, LA 71101. Tel: (318) 212-0248; Fax: (318) 632-6097; Email: oslresearch@aol.com Copyright $\odot$ 2007, Academy of Managed Care Pharmacy. All rights reserved. 
surgery have been reported, including infection, ${ }^{9,10}$ pulmonary embolism, ${ }^{11,12}$ thromboses, ${ }^{11,13-15}$ fat embolism, ${ }^{16}$ hemarthrosis, ${ }^{17}$ patellar fracture, ${ }^{18}$ heterotopic ossification, ${ }^{19,20}$ stiffness, ${ }^{21,22}$ nerve damage, ${ }^{11,23}$ vascular injuries, ${ }^{11,14,24,25}$ and urinary complications. ${ }^{11,12}$ The perioperative mortality rate has been reported at $0.17 \%$ $0.46 \%$ for primary total knee arthroplasty within 90 days $^{26-28}$ and $0.33 \%-0.78 \%$ for bilateral knee replacements within 30 days. ${ }^{28-30}$

Hylan G-F 20 (Synvisc) is a cross-linked hyaluronan derivative approved for the treatment of pain due to OA of the knee after other conservative approaches have failed. Three intra-articular hylan G-F 20 injections effectively relieve the pain associated with $\mathrm{OA}$ of the knee as demonstrated by studies of various design, including double-blind, placebo-controlled; open-label, prospective with or without an active control; and retrospective. ${ }^{31-37}$ In many instances, either medically or by patient choice, delaying the need for a TKR would be advantageous. Previous reports, including a case-control study, a small uncontrolled study, and a theoretical managed care model, suggest that hylan G-F 20 may help delay patient need for TKR. ${ }^{38-40}$

The objective of the current study was to investigate the ability of hylan G-F 20 to delay the need for TKR in patients with predominantly severe OA of the knee in our large orthopedic practice over a 6-year period.

\section{Methods}

\section{Patients}

In our clinical practice, patients diagnosed with OA of the knee who are assessed as clinically suitable candidates for hylan G-F 20 therapy are routinely offered the therapy for OA knee pain relief. We consider a patient with any Kellgren-Lawrence grade of OA without any mechanical problems in the knee (i.e., torn cartilage, severe varus or valgus deformity, or end-stage bone-on-bone disease) as suitable for hylan G-F 20 therapy. We consider a patient a TKR candidate if they have KellgrenLawrence OA grade IV (severe; joint space greatly impaired with sclerosis of subchondral bone) and score on the visual analogue scale (VAS) for pain of approximately $60 \mathrm{~mm}$ or greater (on a 100-mm scale). All patients included in this 6-year retrospective data review were considered TKR candidates when they entered our practice. Instead of being scheduled immediately for knee replacement surgery, all patients received hylan G-F 20. In our practice, patients who are TKR candidates and who are not appropriate for hylan G-F 20 typically undergo surgery within 3 months.

Exclusion criteria for receiving hylan G-F 20 were mechanical symptoms or deformities due to $\mathrm{OA}$, including flexion contracture $>20^{\circ}$, valgus malalignment $>15^{\circ}$, or varus malalignment $>10^{\circ}$. Patients were also not treated if they had any contraindications to the therapy, which are known hypersensitivity to hyaluronan preparations, target knee joint infections, or skin diseases or infections in the area of the injection site. Before undergoing intra-articular hylan G-F 20 injections, all patients had been unsuccessfully treated with nonsteroidal anti-inflammatory drugs and analgesics, and most with COX-2 inhibitors once these were commercially available. At some point, most of the patients had received some form of nonpharmacologic therapy (such as physical therapy) or corticosteroid injections for relief of OA knee pain. Unsuccessful previous treatment was typically defined as a score of 50 on a VAS for pain or as a total score on the Western Ontario McMaster Osteoarthritis Index.

\section{Database Methodology}

Our clinical practice has developed and currently maintains a database of patient information for those receiving hylan G-F 20 for the treatment of OA knee pain. Specific information routinely recorded in the database includes demographic information, osteoarthritic disease characteristics (grade, presence of effusion), prospectively collected data on efficacy measures (e.g., physician VAS for OA knee pain), the occurrence of local adverse events (knee pain and swelling), and the date of any TKR. These prospectively collected data were taken from the records of patients' initial and follow-up visits or follow-up telephone calls if the patient did not return for an office visit.

For this study, data (including TKR status) from the described patients who initiated treatment and/or received repeat treatment with hylan G-F 20 from October 30, 1997, to November 30, 2003, were retrospectively reviewed to explore the ability of hylan G-F 20 to delay patient need for TKR. If TKR status of the patient was unknown as of November 30, 2003, attempts were made to contact the patient by telephone to obtain his or her TKR status.

This study was reviewed retrospectively by an institutional review board, which approved the research protocol. Before treatment, all hylan G-F 20-treated patients signed a standard consent form, which included consent for their health or medical record to be used as a source of data for research.

\section{Treatment With Hylan G-F 20}

Each course of therapy involves 1 intra-articular hylan G-F 20 injection ( $2 \mathrm{~mL}$ ) per week for 3 weeks using a fluoroscopic technique that confirms accurate needle placement in the joint as we have previously described. ${ }^{41}$ The published cost of a course of hylan G-F 20 injections in 2006 was \$852, which includes the cost of 3 injections with arthrocentesis and 1 office visit (Medicare reimbursement policy allows only 1 office visit charge despite the need for 3 visits). ${ }^{42-44}$ The additional cost of fluoroscopy, which is not used by all physicians, is $\$ 77$ per injection or $\$ 231$ per course of therapy.

Patients may have received more than 1 course of therapy during this observation period (Table 1). While product information states that results of repeat use have not been established, ${ }^{45}$ we have published 3 articles on 2 studies indicating the effectiveness and tolerability of repeat courses of hylan G-F $20.46-48$ In our practice, 
we repeat courses of hylan G-F 20 therapy after a patient experiences an initial beneficial response $(\geq 20 \mathrm{~mm}$ reduction in physician VAS for OA knee pain) with the treatment. Subsequent courses of therapy are offered to patients if their VAS score worsens to $50 \%$ of their initial baseline VAS. In our prospective study of a second course of therapy, the mean time between first and second courses was 19 months. ${ }^{46}$

To avoid local adverse events (knee pain and swelling) after injection, we instruct patients to rest the afternoon of the injection, use an ice pack for 2 to 3 hours, and resume normal activities the day after the injection, except for strenuous work or exercise, which should be resumed only after completion of therapy. We also provide patients with a prescription of hydrocodone and acetaminophen, pentazocine hydrogen chloride and acetaminophen, or tramadol hydrogen chloride and acetaminophen, to be used only if they experience postinjection pain and swelling.

\section{Statistical Analysis}

For patients undergoing a TKR during the observation period, median time to TKR was determined. Median time to TKR included treatment time for hylan G-F 20 (2 weeks) and the time up until the TKR was performed. For those patients who did not receive a TKR, median time of patient follow-up was calculated. Median patient follow-up time was defined as the hylan G-F 20 treatment time (2 weeks) plus the time of follow-up after treatment. For patients with known TKR status during the entire observation period, the end of a patient's follow-up time was the end of the observation period (November 30, 2003). Otherwise, the end of a patient's follow-up time was the date of the patient's last office visit if the patient was no longer being seen in our office and could not be contacted by telephone (lost to follow-up). End of follow-up time for deceased patients was their date of death or the date of the last office visit if the exact date of death was not available. If an exact date of the month or year of having a TKR was not available, the first of the indicated month or year was used for the follow-up time calculation.

Because patients with grade IV OA of the knee are considered candidates for TKR in our practice, only patients with grade IV OA of the knee treated with hylan G-F 20 were analyzed. The incidence of TKR in this patient population was calculated. The effects of age, ethnicity, gender, body mass index (BMI), and effusion history at the beginning of hylan G-F 20 treatment on the odds for a TKR were evaluated using logistic regression (the GENMOD procedure in SAS [SAS version 9.1, SAS Institute, Cary, NC]). Age was categorized to 1 of 5 groups: $<50$ years, 50 to 59 years, 60 to 69 years, 70 to 79 years, and $\geq 80$ years. BMI was also categorized into groups: normal $\left(20-25 \mathrm{~kg} / \mathrm{m}^{2}\right)$, overweight $\left(26-30 \mathrm{~kg} / \mathrm{m}^{2}\right)$, obese $\left(31-40 \mathrm{~kg} / \mathrm{m}^{2}\right)$, and severely obese $\left(>40 \mathrm{~kg} / \mathrm{m}^{2}\right)$. If significant covariate effects were detected $(P<0.05)$, covariate effects were evaluated by a Wald chi-square test.

\section{TABLE 1 Disposition of Knee Population During the Observation Period*}

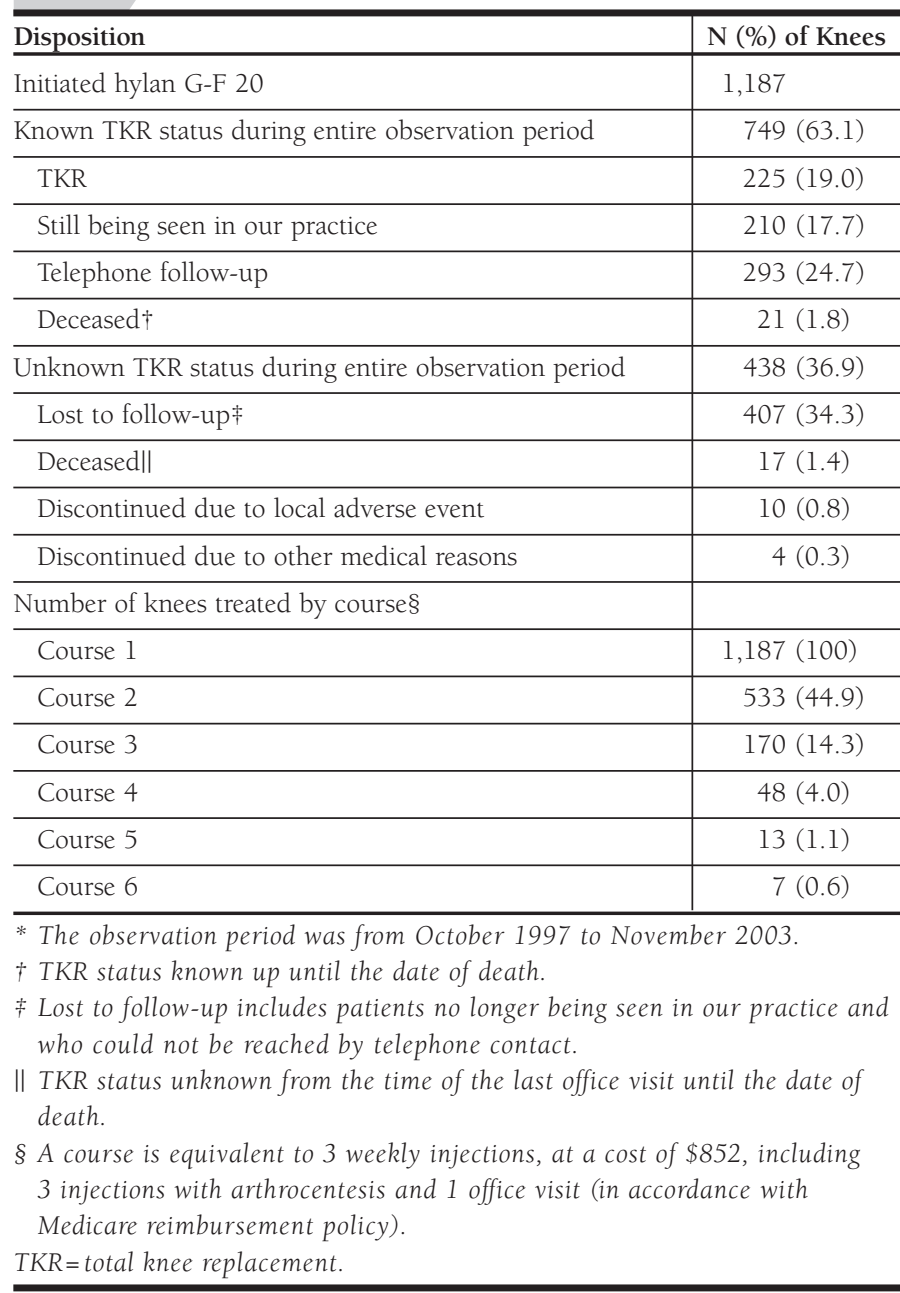

Because not all patients had received a TKR during the observation period, survival analysis was used to evaluate (1) time to TKR for the entire patient population and (2) the effects of age, ethnicity, gender, BMI, and effusion history at the beginning of hylan G-F 20 treatment on the time to TKR. The PHREG procedure in SAS was used to test the effects of these covariates on the time to TKR under the assumption of a proportional hazards model. If significant covariate effects were detected ( $\mathrm{P}<0.05$ ), covariate effects were evaluated by a Wald chisquare test.

\section{Results}

\section{Patient Knee Disposition and Patient Demographics}

A total of 1,187 knees (863 patients) with OA grade IV were treated with hylan G-F 20. The disposition of treated knees, with regard to patient status during the observation period, that 


\begin{tabular}{|c|c|c|}
\hline $\begin{aligned} \text { TABLE 2 } & \mathrm{Pc} \\
& \mathrm{Ta}\end{aligned}$ & $\begin{array}{l}\text { t Demographics aı } \\
\text { Knee Characterist }\end{array}$ & \\
\hline Characteristic & Without TKR $(\mathrm{N}=962)$ & With TKR $(\mathrm{N}=225)$ \\
\hline \multicolumn{3}{|l|}{ Age, years* } \\
\hline Mean \pm SD & $67.5 \pm 13.3$ & $66.8 \pm 10.02$ \\
\hline Range & $28-98$ & $36-89$ \\
\hline \multicolumn{3}{|c|}{ Patient distribution, N (\%) } \\
\hline$<50$ & $109(11.3)$ & $16(7.1)$ \\
\hline $50-59$ & $141(14.7)$ & $31(13.8)$ \\
\hline $60-69$ & $212(22.1)$ & $80(35.7)$ \\
\hline $70-79$ & $343(35.7)$ & $79(35.1)$ \\
\hline$\geq 80$ & $156(16.2)$ & $19(8.4)$ \\
\hline \multicolumn{3}{|l|}{ Gender, N (\%) } \\
\hline Male & $393(40.9)$ & $88(39.1)$ \\
\hline Female & $569(59.1)$ & $137(61.2)$ \\
\hline \multicolumn{3}{|l|}{ Ethnic origin, $\mathrm{N}(\%)$} \\
\hline White & $856(89.0)$ & $207(92.0)$ \\
\hline African American & $106(11.0)$ & $18(8.0)$ \\
\hline \multicolumn{3}{|l|}{ Body mass index, kg/m² } \\
\hline Mean \pm SD & $31.1 \pm 6.6$ & $30.6 \pm 5.6$ \\
\hline Range & $17.9-2.1$ & $19.1-4.1$ \\
\hline \multicolumn{3}{|c|}{ Patient distribution, N (\%) } \\
\hline Normal (20-25) & $140(14.5)$ & $31(31.8)$ \\
\hline Overweight (26-30) & $358(37.2)$ & $78(34.7)$ \\
\hline Obese (31-40) & $370(38.5)$ & $102(45.3)$ \\
\hline Severely obese $(>40)$ & $94(9.8)$ & $14(6.2)$ \\
\hline \multicolumn{3}{|l|}{$\begin{array}{l}\text { History of target knee } \\
\text { effusion, } \mathrm{N}(\%)\end{array}$} \\
\hline No & $822(85.5)$ & $198(88.0)$ \\
\hline Yes & $140(14.6)$ & $27(12.0)$ \\
\hline \multicolumn{3}{|c|}{$\begin{array}{l}\text { Baseline visual analogue } \\
\text { scale for pain, 100-mm scale }\end{array}$} \\
\hline Mean \pm SD & $77.1 \pm 13.6$ & $77.3 \pm 12.4$ \\
\hline
\end{tabular}

were included in the analysis is presented in Table 1 . The number of knees receiving from 1 to 6 courses of therapy is also summarized in Table 1.

The demographic characteristics and TKR status of patients whose knees were treated with hylan G-F 20 are shown in Table 2. The mean age was 67.5 years for patients who did not undergo TKR and 66.8 years for those who underwent TKR. Independent of TKR status, the majority of patients were female and white (the remaining patients were African American), and had above-normal BMI. Most patients had no history of effusion in the target knee.

\section{Incidence and Odds of TKR}

The majority of knees being treated with hylan G-F 20 during the observation time did not require a TKR. A total of 225 knees underwent a TKR, for a TKR incidence of $19 \%$ of knees in this population of surgical candidates with OA grade IV (Table 1). The incidence of TKR by demographic and disease characteristics is shown in Table 3. Within age categories, the incidence of TKR in knees was highest for patients aged 60 to 69 years, which was more than double the percentage of patients aged $<50$ or $\geq 80$ years who had a TKR. Knees from patients who were obese had a higher incidence of TKR compared with knees from patients with normal BMI, or from those who were overweight or severely obese. All other incidences for knees having a TKR within other age categories, gender, ethnicity, and effusion history were comparable with the 19\% incidence for all knees.

Logistic regression showed that of age, gender, ethnicity, $\mathrm{BMI}$, and effusion history, age was the only significant covariate $(P<0.001)$. Odds ratios for age-group comparisons are presented in Table 4 . Knees from patients aged 60 to 69 years were significantly more likely to have had a TKR compared with knees from patients in age groups $<50$ years $(P=0.006), 70$ to 79 years $(P=0.0082)$, and $\geq 80$ years $(P<0.001)$. Patient knees from the $<50$-year-old group were $57 \%$ less likely to have had a TKR than were patient knees from the 60 to 69 -year-old group $(P=0.006)$. Knees of patients in the age groups 50 to 59,60 to 69 , and 70 to 79 years were approximately 2 - to 3 -fold more likely to have had a TKR than were knees of patients $\geq 80$ years $(P<0.05)$.

\section{Time to TKR}

For knees that underwent TKR during the observation period, the median time to TKR following hylan G-F 20 treatment (including 2 weeks for hylan G-F 20 administration) was 1.8 years (638 days; minimum of 14 days, maximum of 2,147 days). For knees in which a TKR had not yet occurred during the observation period, median time of hylan G-F 20 treatment and patient follow-up was 2.2 years (810 days; minimum of 7 days, maximum of 2,222 days). While this latter calculation included patients lost to follow-up, we further calculated the known time of follow-up for knees of patients no longer being seen in the practice (lost to follow-up): 1.1 years (416 days; minimum of 7 days, maximum of 2,112 days).

Collectively, the median time of all knees to either TKR or time of last observation was 2.1 years (772 days; minimum 7 , maximum 2,222). Based on the current cost of hylan G-F 20 (\$852, including 3 injections with arthrocentesis and 1 office visit [in accordance with Medicare reimbursement policy]), we calculated that 1,978 courses of hylan G-F 20 given to 1,187 knees (average 1.67 course per knee) resulted in an average cost of $\$ 1,419.76$ per knee to delay TKR by a median of 2.1 years (median time for all knees).

Survival analysis of all knees for time to TKR is shown in Figure 1, which indicates that $75 \%$ of knees had not had a TKR 
by 3.8 years ( 1,370 days; $95 \%$ confidence interval [CI], 1,215-1,590 days). These analyses included data on those lost to follow-up who had not had a TKR at the time of their last office visit.

\section{Effect of Demographic Variables on Time to TKR}

Of the covariates tested in the survival analysis (age, gender, ethnicity, BMI, and effusion history), age was the only significant predictor for time to TKR $(P=0.009)$. Figure 2 illustrates the survival analysis for time to TKR by age group. Knees of patients in the age group 60 to 69 years had the shortest amount of time to TKR, while knees of patients aged $<50$ years had the longest time to TKR, followed by knees of patients aged $\geq 80$ years (Figure 2 ). Time to TKR was significantly shorter for knees of patients in the 60 to 69 years age group compared with knees of patients aged $<50$ years $(P=0.003)$ and of patients aged $\geq 80$ years $(P<0.004)$. For patients aged 70 to 79 years, time to TKR was significantly shorter than for patients aged $<50$ years $(P=0.033)$ and patients aged $\geq 80$ years $(P=0.047)$. No other age comparisons were significantly different.

\section{Discussion}

This study suggests that the use of 1 or more courses of hylan G-F 20 in orthopedic practice can delay the need for surgery in patients who are candidates for TKR (OA grade IV knees). Delaying TKR can be advantageous for patients who do not want surgery or for whom surgery is not medically desirable. The incidence of TKR in the population of TKR candidates studied here was low, and for knees that underwent TKR, use of hylan G-F 20 had the ability to delay surgery for approximately 1.8 years. In others, the median time of hylan G-F 20 treatment and patient follow-up without having a TKR was 2.2 years.

Previously reported data also show that hylan G-F 20 can delay the need for TKR in patient populations with predominantly advanced OA. In an open-label, multicenter trial, OA knee pain in $86 \%$ of patients $(n=60)$ with advanced OA $(75 \%$ grade IV) receiving hylan G-F 20 therapy (up to 4 courses) improved sufficiently enough to delay TKR at week 12 of the study. ${ }^{39}$ At the end of the 30-month observation period, a total of 59\% of treated patients were able to delay TKR. ${ }^{39}$ We reported a preliminary, case-control study (110 TKR patients; 1,151 patients without TKR) in which the probability of progression to TKR in a population of predominantly advanced OA (83\% grade IV) knee patients was reduced with hylan G-F 20 therapy.

Although TKR has been shown to be an effective therapy for patients ranging in age from $<50$ years to $>80$ and 90 years, ${ }^{40-51}$ patients aged $<50$ years or $>80$ years may not be the best TKR candidates and are thus less likely to undergo the procedure. Our study reflects this trend because we found that patients aged $<50$ years and patients aged $\geq 80$ years were significantly less likely to have had a TKR than other age groups. For example, patients aged $<50$ years were significantly less likely to have had a TKR than 60 to 69 -year-olds, and patients aged $\geq 80$ years

\section{TABLE 3} Incidence of Total Knee Replacement by Demographic and Disease Characteristics

\begin{tabular}{|c|c|c|}
\hline Characteristic & $\begin{array}{l}\text { Patients per } \\
\text { Subgroup, N }\end{array}$ & $\begin{array}{c}\text { TKR Incidence, } \\
\text { N (\%) }\end{array}$ \\
\hline \multicolumn{3}{|l|}{ Age distribution, years } \\
\hline$<50$ & 125 & $16(12.8)$ \\
\hline $50-59$ & 172 & $31(18.0)$ \\
\hline $60-69$ & 292 & $80(27.4)$ \\
\hline $70-79$ & 422 & $79(18.7)$ \\
\hline$\geq 80$ & 175 & $19(10.9)$ \\
\hline \multicolumn{3}{|l|}{ Gender } \\
\hline Male & 481 & $88(18.3)$ \\
\hline Female & 706 & $137(19.4)$ \\
\hline \multicolumn{3}{|l|}{ Ethnic origin } \\
\hline White & 1,063 & $207(19.5)$ \\
\hline African American & 124 & $18(14.5)$ \\
\hline \multicolumn{3}{|c|}{ Body mass index distribution, $\mathrm{kg} / \mathrm{m}^{2}$} \\
\hline Normal (20-25) & 171 & $31(18.1)$ \\
\hline Overweight (26-30) & 436 & $78(17.9)$ \\
\hline Obese (31-40) & 472 & $102(21.6)$ \\
\hline Severely obese $(>40)$ & 108 & $14(13.0)$ \\
\hline \multicolumn{3}{|c|}{ History of target knee effusion } \\
\hline No & 1,020 & $198(19.4)$ \\
\hline Yes & 167 & $27(16.2)$ \\
\hline
\end{tabular}

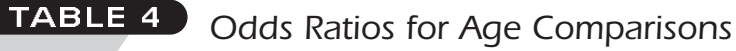 With Logistic Regression}

\begin{tabular}{l|c|c|c}
\hline Age, years & Odds Ratio & $95 \%$ CI & $P$ Value \\
\hline$<50$ vs. $50-59$ & 0.65 & $0.34-1.27$ & 0.2104 \\
\hline$<50$ vs. $60-69$ & 0.43 & $0.24-0.79$ & 0.0060 \\
\hline$<50$ vs. $70-79$ & 0.70 & $0.38-1.28$ & 0.2489 \\
\hline$<50$ vs. $<80$ & 1.35 & $0.64-2.85$ & 0.4289 \\
\hline $50-59$ vs. $60-69$ & 0.66 & $0.41-1.06$ & 0.0853 \\
\hline $50-59$ vs. $70-79$ & 1.07 & $0.66-1.73$ & 0.7729 \\
\hline $50-59$ vs. $\geq 80$ & 2.07 & $1.08-3.96$ & 0.0282 \\
\hline $60-69$ vs. $70-79$ & 1.63 & $1.14-2.35$ & 0.0082 \\
\hline $60-69$ vs. $\geq 80$ & 3.15 & $1.79-5.53$ & $<0.0001$ \\
\hline $70-79$ vs. $\geq 80$ & 1.93 & $1.12-3.32$ & 0.0183 \\
\hline
\end{tabular}

* Wald chi-square test.

$C I=$ confidence interval. 


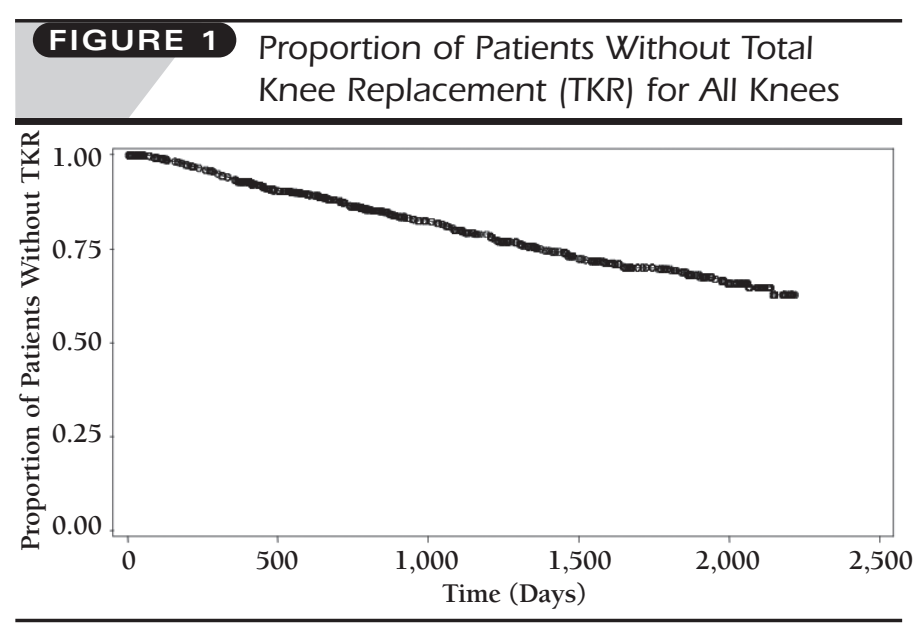

Candidates for TKR entering our practice who are not appropriate for hylan G-F 20 typically undergo surgery within 3 months.

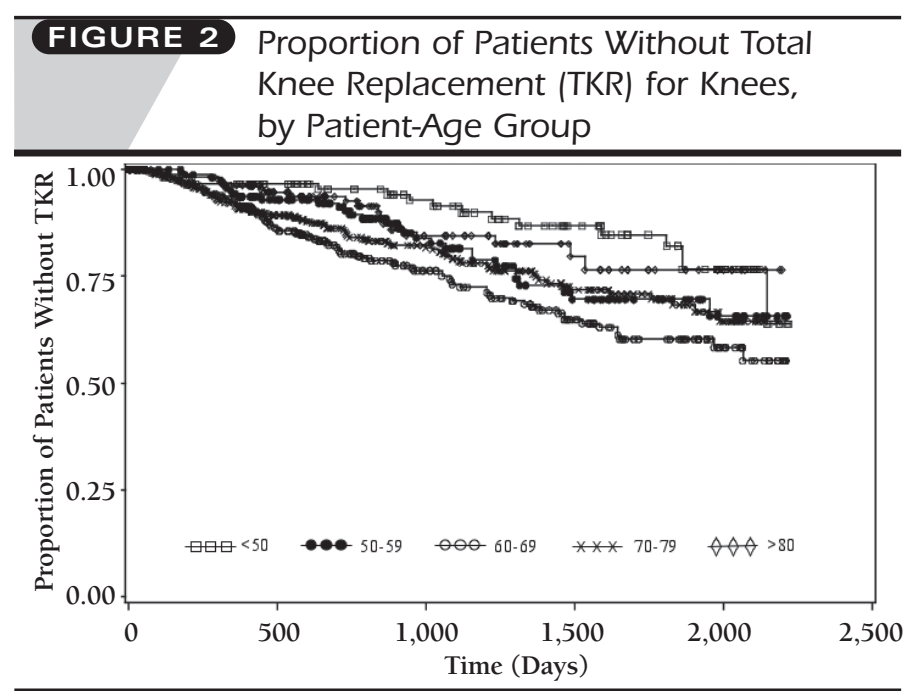

Numbers in the legend indicate age (years).

were significantly less likely to have had a TKR than patients aged 50 to 59 years, 60 to 69 years, and 70 to 79 years. Patients in the age group 60 to 69 years had the highest incidence of TKR and the shortest time to TKR compared with the other age groups. Survival analysis also showed that patients aged $<50$ years and $\geq 80$ years had the longest time to TKR, reflecting inclusion of data for patients lost to follow-up who had not had a TKR as of their last office visit.

In many cases, it may be desirable to delay TKR in younger patients because of the risk of hardware revisions, loosening from the bone, pain from overuse, or to delay or avoid the procedure altogether in older patients or patients with comorbidities who may increase their complication risk with surgery.
Therefore, delaying TKR by incorporating hylan G-F 20 into the treatment plans for these patients is advantageous. Avoiding surgical treatment might also prevent complications that can arise from TKR, including, but not limited to, infection, ${ }^{9,10}$ pulmonary embolism, ${ }^{11,12}$ thromboses, ${ }^{11,13-15}$ fat embolism, ${ }^{16}$ hemarthrosis, ${ }^{17}$ patellar fracture, ${ }^{18}$ heterotropic ossification, ${ }^{19,20}$ stiffness, ${ }^{21,22}$ nerve damage, ${ }^{11,23}$ vascular injuries, ${ }^{11,14,24,25}$ urinary complications, ${ }^{11,12}$ and even death for some. ${ }^{26-28}$ In addition, risk for complication in an older patient due to the delay in TKR is dependent on individual patient factors. The most common adverse events that occur with hylan G-F 20 are local knee pain and/or swelling, which have occurred in $2.2 \%$ of injections or in $7.2 \%$ of patients treated with hylan G-F $20 .{ }^{45}$ We have reported a similar low incidence of local adverse events from the same database of this report (1.2\% of injections, $5.2 \%$ of patients)..$^{52}$ If local knee pain and swelling do occur with hylan G-F 20, it is transient and clinically manageable ${ }^{53}$ and is less serious than most of the surgical complications listed above. No serious systemic adverse events have been observed with hylan G-F 20 in clinical trials. 45

With the estimated increase in the $\geq 65$-year-old population with $\mathrm{OA}$ in years to come, strategies that would help alleviate the economic burden of OA, including TKR, would be appropriate for providers and policymakers. Given that TKRs are the primary cost drivers for $\mathrm{OA}$ of the knee, cost savings to any plan would be a function of delaying or avoiding the number of TKRs in their covered patients. ${ }^{40}$ With its ability to delay TKR, use of hylan G-F 20 therapy has the potential to reduce costs for $\mathrm{OA}$ knee treatment for not only surgery but revision surgery. A theoretical managed care model with a large Medicare population developed to evaluate the potential savings associated with incorporating hylan G-F 20 into the standard treatment regimen for OA knee pain demonstrated cost savings. ${ }^{40}$ In this hypothetical cohort of 100,000 patients (3,835 with mild, moderate, or severe OA of the knee) followed for 3 years, significant cost savings were predicted with the use of hylan G-F $20{ }^{40}$ This cost savings was primarily due to the predicted 808 TKRs that could be avoided with hylan G-F 20 therapy. ${ }^{40}$ Over this 3-year period, a total cost savings of $\$ 8,810,771$ was reported for the plan, with a total cost savings of $\$ 4,706$ per patient receiving hylan G-F $20 .^{40}$

The results of this theoretical model in conjunction with the clinical reports above $e^{38,39}$ and our clinical experience reported here support significant cost savings with hylan G-F 20 for the OA knee patient as well as for insurance and managed care plans. On the basis of the current cost of hylan G-F 20 (\$852, including 3 injections with arthroscopy and 1 office visit (in accordance with Medicare reimbursement policy), we calculated that 1,978 courses of hylan G-F 20 given to 1,187 knees resulted in an average cost of $\$ 1,419.76$ per knee to delay TKR by a median of 2.1 years, the median time of all knees to either TKR or time of last observation. 


\section{Limitations}

First, the current study is limited as a retrospective review. Second, since this is a review of a database of only patients who were treated with hylan G-F 20, there is no control group. Without a control group, the effect of the injection itself as well as other patient factors (medical or otherwise) could not be controlled. Candidates for TKR who enter our practice and are not treated with hylan G-F 20 because they are not good candidates for the therapy typically undergo surgery within 3 months. This patient group would be the closest comparison to a control group in our practice. In addition to the fact that these patients are different from those treated with hylan G-F 20 because they are not good hylan G-F 20 candidates, there are other reasons patients may delay surgery (e.g., patient choice, office scheduling). However, our typical patients who require a TKR (without hylan G-F 20 therapy and having surgery within 3 months) are a realistic comparison given all other factors. Furthermore, many of the patients were satisfied enough with therapy that they pursued more than 1 course of hylan G-F 20 treatment. Our interpretation of the data presented here is that hylan G-F 20 therapy may reduce the OA of the knee of TKR candidates enough to delay surgery. We found that this delay was approximately 2 years following hylan G-F 20 treatment.

Third, in the present study, hylan G-F 20 was administered using a fluoroscopic technique that confirms accurate needle placement in the joint. Fluoroscopy is one of a number of measures that can be used to ensure proper placement of hylan G-F 20 in the intra-articular joint space and is on the same level of standard of care as joint aspiration. Without using fluoroscopy, there may be a higher chance of inaccurate needle placement, which is thought to be a cause for local pain and swelling following injection. Practices that do not use fluoroscopy to administer hylan G-F 20 may have results that differ from our study.

Fourth, this report is also limited by the lack of knowledge of patient outcome with regard to TKR after the time patients were treated and seen in our office. Including these patients in the calculation for treatment and follow-up time, we found the time of TKR delay was at least 2.2 years. Although the number of patients lost to follow-up (34\%), likely because of normal attrition, was quite high, eliminating these patients from the analysis would introduce more bias into the study. To address the lack of knowing patient outcome with regard to TKR in these patients, we calculated their time of hylan G-F 20 treatment and follow-up based on their last office visit or last telephone contact. We found their time of treatment and followup to be at least 1 year. So despite not knowing their outcome after our last contact with those patients, we found their surgery was still delayed past the 3-month timing it takes for the typical patient requiring a TKR to undergo the procedure in our practice. The strengths of this study lie in the high patient number
(1,187 knees of 863 patients) and the length of the observation period that spanned 6 years.

\section{Conclusion}

This study suggests that hylan G-F 20 can delay patient need for TKR in a population of OA knee patients with advanced disease. The ability of hylan G-F 20 to delay TKR is advantageous for patients for whom TKR is not medically appropriate or for patients who do not want or who fear surgery. In select patients, delaying TKR will also reduce the risk of any potential complications that may accompany the procedure. As long as TKR candidates can maintain a functional lifestyle and good quality of life with an alternative therapy, delaying TKR is beneficial. Further implications may be made with regard to cost savings for patients, policymakers, and managed care and insurance plans.

\section{What is already known about this subject}

- In patients failing usual therapy, TKR may be medically contraindicated due to comorbidities, or is not preferred by some patients, as a last-resort treatment for $\mathrm{OA}$ of the knee.

- Hylan G-F 20 is approved for the treatment of pain due to OA of the knee.

\section{What this study adds}

- One or more courses of hylan G-F 20 for the treatment of grade IV OA of the knee may delay a patient's need for TKR.

\section{ACKNOWLEDGMENTS}

The authors would like to thank Brenda Garlington and Brad S. Waddell for their expert research assistance; Steven V. Radecki, PhD, Fort Collins, Colorado, for statistical analysis; Kathleen M. Ohleth, PhD, Precise Publications, LLC, Pluckemin, New Jersey, for manuscript preparation; and for support provided to Radecki and Ohleth by Wyeth Pharmaceuticals and Genzyme Biosurgery.

\section{DISCLOSURES}

Funding for manuscript preparation and statistical analysis was provided by Wyeth Pharmaceuticals and Genzyme Biosurgery and was sought by author David Waddell. Waddell was a consultant to Wyeth Pharmaceuticals at the time of this study and is currently a consultant to Genzyme Biosurgery; author DeWayne C. Bricker has participated in Wyeth's speakers bureau.

Waddell served as principal author of the study. Study concept and design and writing of the manuscript and its revision were the work of Waddell. Data collection was primarily the work of Waddell, with input from Bricker; data interpretation was the work of Waddell.

\section{REFERENCES}

1. U.S. Dept. of Health \& Human Services, Centers for Disease Control and Prevention. Prevalence of disabilities and associated health conditions among adults-United States, 1999. MMWR. 2001;50:120-25.

2. Srikanth VK, Fryer JL, Zhai G, Winzenberg TM, Hosmer D, Jones G. A meta-analysis of sex differences prevalence, incidence, and severity of osteoarthritis. Osteoarthritis Cartilage. 2005;13:769-81. 
3. Gupta S, Hawker GA, Laporte A, Croxford R, Coyte PC. The economic burden of disabling hip and knee osteoarthritis $(\mathrm{OA})$ from the perspective of individuals living with this condition. Rheumatology (Oxford). 2005; 44:1531-37.

4. Altman RD, Hochberg MC, Moskowitz RW, Schnitzer TJ. Recommendations for the medical management of osteoarthritis of the hip and knee. Arthritis Rheum. 2000;43:1905-15.

5. Kozak LJ, Owings MF, Hall MJ. National Hospital Discharge Survey: 2001 annual summary with detailed diagnosis and procedure data. National Center for Health Statistics. Vital Health Stat. 2004;13.

6. Melzer D, Guralnik JM, Brock D. Prevalence and distribution of hip and knee joint replacements and hip implants in older Americans by the end of life. Aging Clin Exp Res. 2003;15:60-66.

7. The Institute of Orthopaedic Enlightenment. The Ortho FactBook. 2002-2003 Series, U.S. 3rd ed. Chagrin Falls, OH: Knowledge Enterprises, Inc.; 2002.

8. National Institutes of Health. NIH consensus statement on total knee replacement, December 8-10, 2003. J Bone Joint Surg Am. 2004;86-A:1328-35.

9. Buechel FF. The infected total knee arthroplasty: just when you thought it was over. J Arthroplasty. 2004;19(suppl 1):51-55.

10. Segawa H, Tsukayama DT, Kyle RF, Becker DA, Gustilo RB. Infection after total knee arthroplasty. A retrospective study of the treatment of eighty-one infections. J Bone Joint Surg Am. 1999;81-A:1434-45.

11. Lombardi AV, Mallory TH, Fada RA, et al. Simultaneous bilateral total knee arthroplasties: who decides? Clin Orthop Rel Res. 2001;392:319-29.

12. Weaver F, Hynes D, Hopkinson W, et al. Preoperative risks and outcomes of hip and knee arthroplasty in the Veterans Health Administration. J Arthroplasty. 2003;18:693-708.

13. Ginsberg JS, Turkstra F, Buller HR, Mackinnon B, Magier D, Hirsh J. Postthrombotic syndrome after hip or knee arthroplasty: a cross-sectional study. Arch Intern Med. 2000;160:669-72.

14. Saleh KJ, Hoeffel DP, Kassim RA, Burstein G. Complications after revision total knee arthroplasty. J Bone Joint Surg Am. 2003;85-A(suppl 1):S71-S74.

15. Kim YH, Oh SH, Kim JS. Incidence and natural history of deep-vein thrombosis after total hip arthroplasty. A prospective and randomised clinical study. J Bone Joint Surg Br. 2003;85-B:661-65.

16. Kim YH. Incidence of fat embolism syndrome after cemented or cementless bilateral simultaneous and unilateral total knee arthroplasty. J Arthroplasty. 2001;16:730-39.

17. Ohdera T, Tokunaga M, Hiroshima S, Yoshimoto E, Matsuda S. Recurrent hemarthrosis after knee joint arthroplasty: etiology and treatment. J Arthroplasty. 2004;19:157-61.

18. Ortiguera CJ, Berry DJ. Patellar fracture after total knee arthroplasty. J Bone Joint Surg Am. 2002;84-A:532-40.

19. Dalury DF, Jiranek WA. The incidence of heterotopic ossification after total knee arthroplasty. J Arthroplasty. 2004;19(suppl 1):447-52.

20. Toyoda T, Matsumoto H, Tsuji T, Kinouchi J, Fujikawa K. Heterotopic ossification after total knee arthroplasty. J Arthroplasty. 2003;18:760-64.

21. Laskin RS, Beksac B. Stiffness after total knee arthroplasty. J Arthroplasty. 2004;19(suppl 2):41-46.

22. Bong MR, Di Cesare PE. Stiffness after total knee arthroplasty. J Am Acad Orthop Surg. 2004;12:164-71.

23. Idusuyi OB, Morrey BF. Peroneal nerve palsy after total knee arthroplasty. Assessment of predisposing and prognostic factors. J Bone Joint Surg Am. 1996;78-A:177-84

24. Da Silva MS, Sobel M, The Surgeons of the Southern Association of Vascular Surgery. Popliteal vascular injury during total knee arthroplasty. J Surg Res. 2003;109:170-74.

25. Langkamer VG. Local vascular complications after knee replacement: a review with illustrative case reports. Knee. 2001;8:259-64.
26. Bhattacharyya T, Iorio R, Healy WL. Rate of and risk factors for acute inpatient mortality after orthopaedic surgery. J Bone Joint Surg. 2002;84A:562-72.

27. Gill GS, Mills D, Joshi AB. Mortality following primary total knee arthroplasty. J Bone Joint Surg Am. 2003;85-A:432-35.

28. Parvizi J, Sullivan TA, Trousdale RT, Lewallen DG. Thirty-day mortality after total knee arthroplasty. J Bone Joint Surg Am. 2001;83-A:1157-61.

29. Nunley RM, Lachiewicz PF. Mortality after total hip and knee arthroplasty in a medium-volume university practice. J Arthroplasty. 2003;18:278-85.

30. Bullock DP, Sporer SM, Shirreffs TG. Comparison of simultaneous bilateral with unilateral total knee arthroplasty in terms of perioperative complications. J Bone Joint Surg. 2003;85-A:1981-86.

31. Wobig M, Dickhut A, Maier R, Vetter G. Viscosupplementation with hylan G-F 20: a 26-week controlled trial of efficacy and safety in the osteoarthritic knee. Clin Ther. 1998;20:410-23.

32. Scale D, Wobig M, Wolpert W. Viscosupplementation of osteoarthritic knees with hylan: a treatment schedule study. Curr Ther Res. 1994;55:22032.

33. Adams ME. An analysis of clinical studies of the use of crosslinked hyaluronan, hylan, in the treatment of osteoarthritis. J Rheumatol. 1993;20: 16-18.

34. Adams ME, Atkinson MH, Lussier AJ, et al. The role of viscosupplementation with hylan G-F 20 (Synvisc) in the treatment of osteoarthritis of the knee: a Canadian multicenter trial comparing hylan G-F 20 alone, hylan G-F 20 with non-steroidal anti-inflammatory drugs (NSAIDs) and NSAIDs alone. Osteoarthritis Cartilage. 1995;3:213-25.

35. Raynauld JP, Torrance GW, Band PA, et al. A prospective, randomized, pragmatic, health outcomes trial evaluating the incorporation of hylan G-F 20 into the treatment paradigm for patients with knee osteoarthritis (part 1 of 2): clinical results. Osteoarthritis Cartilage. 2002;10:506-17.

36. Wobig M, Beks P, Dickhut A, Maier R, Vetter G. Open-label multicenter trial of the safety and efficacy of viscosupplementation with hylan G-F 20 (Synvisc) in primary osteoarthritis of the knee. J Clin Rheumatol. 1999;5:S24-S31.

37. Lussier A, Cividino AA, McFarlane CA, Olszynski WP, Potashner WJ, De Medicis R. Viscosupplementation with hylan for the treatment of osteoarthritis: findings from clinical practice in Canada. J Rheumatol. 1996;23:1579-85.

38. Waddell DD, Bricker D. Hylan G-F 20 reduces the probability of progression to total knee replacement in knee osteoarthritis. Arthritis Rheum. 2001; 44:S49.

39. Bell M, Fallaha M, Lenczner E, Ranger P, Welsh P. Viscosupplementation with hylan G-F 20 in total knee replacement candidates: an effective pain management therapy that may delay surgery. Osteoarthritis Cartilage. 1999;7 (suppl A):S30

40. Waddell D, Rein A, Panarites C, Coleman M, Weiss C. Cost implications of introducing an alternative treatment for patients with osteoarthritis of the knee in a managed care setting. Am J Manag Care. 2001;7:981-91.

41. Waddell D, Estey D, Bricker DC, Marsala A. Viscosupplementation under fluoroscopic control. Am J Med Sports. 2001;4:237-241,249.

42. Drug Topics Red Book [CD-ROM]. November 2006. Montvale, NJ: Medical Economics.

43. National average physician reimbursement rates. Federal Register. 2006; 70:223.

44. U.S. Dept. of Health \& Human Services, Centers for Medicare \& Medicaid Services. October 2006 ASP pricing file. Available at: http://www.cms.hhs.gov/ McrPartBDrugAvgSalesPrice/02_aspfiles.asp. Accessed October 19, 2006.

45. Synvisc (hylan G-F 20) [product information]. Ridgefield, NJ: Genzyme Biosurgery; 2003. 
46. Waddell DD, Cefalu CA, Bricker DC. An open-label study of a second course of hylan G-F 20 for the treatment of pain associated with knee osteoarthritis. Curr Med Res and Opin. 2003;19:499-507.

47. Waddell DD, Cefalu CA, Bricker DC. A second course of hylan G-F 20 for the treatment of osteoarthritic knee pain: 12-month patient follow-up. J Knee Surg. 2005;18:7-15.

48. Waddell DD, Bricker DC. Hylan G-F 20 tolerability with repeat treatment in a large orthopedic practice: a retrospective review. J Surg Orthop Adv. 2006;15:53-59

49. Mont MA, Lee CW, Sheldon M, Lennon WC, Hungerford DS. Total knee arthroplasty in patients $\leq 50$ years old. J Arthroplasty. 2002;17:538-43.
50. Brander VA, Malhotra S, Jet J, Heinemann AW, Stulberg SD. Outcomes of hip and knee arthroplasty in persons aged 80 years and older. Clin Orthop Rel Res. 1997;345:67-78.

51. Pagnano MW, McLamb LA, Trousdale RT. Total knee arthroplasty for patients 90 years of age and older. Clin Orthop. 2004;179-83.

52. Waddell DD, Bricker D. Clinical experience with the effectiveness and tolerability of hylan G-F 20 in 1047 patients with osteoarthritis of the knee. J Knee Surg. 2006;19:19-27.

53. Waddell DD. The tolerability of viscosupplementation: low incidence and clinical management of local adverse events. Curr Med Res Opin. 2003;19: P1-P6. 